\title{
Magnetic resonance volumetry and spectroscopy of hippocampus and insula in relation to severe exposure of traumatic stress
}

\author{
CINDY ECKART, ${ }^{\mathrm{a}, \mathrm{d}} \mathrm{JÖRN}$ KAUFMANN, ${ }^{\mathrm{b}}$ MARTIN KANOWSKI, ${ }^{\mathrm{b}}$ CLAUS TEMPELMANN, ${ }^{\mathrm{b}}$ \\ HERMANN HINRICHS, ${ }^{\mathrm{b}}$ THOMAS ELBERT, ${ }^{\mathrm{a}}$ HANS-JOCHEN HEINZE, ${ }^{\mathrm{b}}$ AND IRIS-TATJANA KOLASSA ${ }^{\text {a.c.e }}$ \\ aClinical Psychology \& Neuropsychology, Department of Psychology, University of Konstanz, Konstanz, Germany \\ 'University Department of Neurology, University Medical Center Magdeburg, Magdeburg, Germany \\ 'Zukunftskolleg, University of Konstanz, Konstanz, Germany \\ ${ }^{\mathrm{d} D e p a r t m e n t ~ o f ~ S y s t e m s ~ N e u r o s c i e n c e, ~ U n i v e r s i t y ~ M e d i c a l ~ C e n t e r ~ H a m b u r g-E p p e n d o r f, ~ H a m b u r g, ~ G e r m a n y ~}$ \\ 'Department of Clinical \& Biological Psychology, Institute of Psychology and Education, University of Ulm, Ulm, Germany
}

\begin{abstract}
Severe and chronic stress affects the hippocampus, especially during development. However, studies concerning structural alterations of the hippocampus yielded a rather inconsistent picture. Moreover, further anxiety-relevant brain regions, such as the insula, might be implicated in the pathophysiology of posttraumatic stress disorder (PTSD). We combined magnetic resonance (MR) volumetric and spectroscopic analyses of hippocampus and insula in highly traumatized refugees without a history of alcohol/substance abuse or other comorbid diseases. No PTSD-related difference was apparent in the volumes or neurometabolite levels of bilateral hippocampus or insula. However, an association between left hippocampal $\mathrm{N}$-acetyl-aspartate (NAA) and adverse childhood experiences indicated a potential detrimental effect of the early environment on hippocampal integrity. Our results add to increasing evidence that PTSD-related, morphological alterations in the hippocampus are a consequence of early adversity or may result from other factors, such as extensive use of alcohol.
\end{abstract}

Descriptors: Posttraumatic stress disorder, Hippocampus, Insula, Volumetry, MR spectroscopy

A prominent model of posttraumatic stress disorder (PTSD) attributes its symptoms to an exaggerated amygdaloid reactivity that cannot be adequately shaped by the medial prefrontal cortex and the hippocampus (Elbert \& Schauer, 2002; Kolassa \& Elbert, 2007). Analogous to findings of stress-induced hippocampal cell loss in animals (Sapolsky, Uno, Rebert, \& Finch, 1990), it was supposed that atrophic processes might be responsible for this impairment. Indeed, PTSD-related volume reductions were reported for the whole hippocampus (Gilbertson et al., 2002; Gurvits et al., 1996; Lindauer et al., 2004; Schmahl et al., 2009; Shin et al., 2004), its body (Bremner et al., 2003), its tail (Bonne et al., 2008), and its head (Vythilingam et al., 2005) - a pattern that, however, has not consistently been replicated (Agartz, Momenan, Rawlings, Kerich, \& Hommer, 1999; Bonne et al., 2001; De Bellis, Hall, Boring, Frustaci, \& Moritz, 2001; Golier et al., 2005; Jatzko et al., 2006; Schuff et al., 2001, 2008; Wood-

We would like to thank Dawan Ali Mohammad, who supported the recruitment of the subjects, and Kathrin Zierhut, who supported the hippocampal volumetry by affording a second independent rating for some of the subjects. This research was supported by the German Research Foundation (DFG). This research was funded by the DFG research unit Sciences of Social Stress (SOSS, DFG-grant EL 101/24), the Zukunftskolleg of the University of Konstanz, and the Center of Advanced Imaging Magdeburg (CAI, BMBF-grant 01GO0504).

Address correspondence to: Cindy Eckart, University Medical Center Hamburg-Eppendorf, Martinistr. 52, 20246 Hamburg, Germany. E-mail: c.eckart@uke.de ward et al., 2006). In order to explain some of these inconsistencies, it has been speculated that potential brain atrophies in PTSD might be too subtle to be confidently revealed with volumetric measures (Karl \& Werner, 2009). Accordingly, increasing interest has been directed towards alternative techniques such as magnetic resonance spectroscopy (MRS), which aims to reveal modified levels of several brain metabolites, for example, N-acetylaspartate (NAA). Still, the finding of PTSD-related reductions in hippocampal NAA/creatine levels (Freeman, Cardwell, Karson, \& Komoroski, 1998; Mohanakrishnan Menon, Nasrallah, Lyons, Scott, \& Liberto, 2003) or absolute NAA concentrations (Schuff et al., 2001, 2008) was not confirmed in all studies (Freeman et al., 2006; Geuze, Vermetten, \& Bremner, 2005a). Accordingly, the method of measurement cannot be the only reason for diverging results within the literature.

Another possible methodological issue that has been discussed as a confounding factor in PTSD-related brain research is the high occurrence of comorbid psychiatric disorders that are associated with hippocampal volume reductions by themselves - as, for example, borderline personality disorder (Driessen et al., 2000) or alcohol abuse (Agartz et al., 1999; Woodward et al., 2006). Accordingly, the frequent incidence particularly of alcohol abuse in many of the populations under investigation (e.g., Bremner et al., 2003; Gilbertson et al., 2002; Schuff et al., 2001; Vythilingam et al., 2005) makes it difficult to unequivocally identify the consequences of traumatization and/or PTSD on hippocampal integrity. 
Overall, PTSD-related structural brain research has so far mainly concentrated on amygdala, hippocampus, and medial prefrontal cortex. However, it has repeatedly been stated that this model cannot entirely account for the complex symptom pattern associated with PTSD (e.g., Liberzon \& Martis, 2006). The insula for example, possesses connections to all structures implicated in PTSD (Augustine, 1996) and interacts with them during contextual fear conditioning in humans (Alvarez, Biggs, Chen, Pine, \& Grillon, 2008). Furthermore, an important role of this structure in the formation of emotions (Craig, 2002, 2009) and the recall/ imagery of emotional conditions (Phan, Wager, Taylor, \& Liberzon, 2002; Rasch et al., 2009) is well known. Thus, it has recently been suggested that the insula might play a specific role in the development of PTSD symptoms (Liberzon \& Martis, 2006). Indeed, a stronger functional connectivity between insula and amygdala was, for example, reported in carriers of a deletion variant of ADRA2B, the gene encoding the $\alpha 2 \mathrm{~b}$-adrenergic receptor (Rasch et al., 2009) — a variant that has also been associated with enhanced traumatic memory in survivors of the Rwandan genocide (de Quervain et al., 2007). Moreover, the activity in the insula correlated with the intensity of PTSD-related intrusive memories (Osuch et al., 2001). Taken together, reports about disturbed insula activity in PTSD patients are numerous (Etkin \& Wager, 2007; Kolassa et al., 2007; Liberzon \& Martis, 2006). However, it still remains to be clarified whether these functional alterations are associated with macroscopic structural alterations in this region as well.

To resolve some of the questions raised above, we combined MR volumetry and MRS in the hippocampus and insula of highly traumatized refugees with and without PTSD in comparison to nonstressed controls. The investigated sample had no history of alcohol/substance abuse or other psychiatric disorders. We expected structural alterations in both brain structures (at least in the neurometabolite levels) that could thus unequivocally be attributed to traumatic stress and/or PTSD. Furthermore, in an attempt to identify relevant factors for these alterations, we associated the brain measures to the exposure to traumatic stressors (in adulthood or childhood) and PTSD symptom severity. Finally, we associated hippocampal brain measures with the performance in a spatial memory task, as we expected hippocampal dysfunction on the behavioral level as well.

\section{Materials and Methods}

\section{Setting and Subjects}

Subjects were recruited from local shelters for asylum seekers and Kurdish recreational facilities in Germany. Participants were included if they were male refugees between the ages of 18 to 55 years. Exclusion criteria were (a) psychiatric conditions other than PTSD or major depression, (b) lifetime alcohol and/or substance abuse or dependence, (c) any severe physical impairment (e.g., neurological diseases), and (d) any contraindication for magnetic resonance imaging (MRI). Depressive symptoms are highly comorbid with PTSD and thus were not exclusion criteria because this would have led to an atypical sample of PTSD patients (O'Donnell, Creamer, \& Pattison, 2004). Fifty-two refugees participated in the study. Five subjects ( 2 controls and 3 traumatized controls) were excluded because they aborted the MR scan at the beginning of the spectroscopy. Participants who completed some but not all spectroscopic scans remained in the analysis for those scans they did complete. Thus, 47 refugees entered the final analysis: 20 participants currently suffering from PTSD, 16 traumatized
Table 1. Traumatization and PTSD Symptoms

\begin{tabular}{|c|c|c|c|c|c|c|}
\hline & \multicolumn{2}{|c|}{$\begin{array}{c}\text { Traumatized } \\
\text { controls }\end{array}$} & \multicolumn{2}{|c|}{$\begin{array}{c}\text { PTSD } \\
\text { patients }\end{array}$} & \multirow{2}{*}{$\begin{array}{c}\text { Kruskal-Wallis } \\
\chi^{2}(1)\end{array}$} & \multirow[b]{2}{*}{$p$ values } \\
\hline & $M$ & $S D$ & $M$ & $S D$ & & \\
\hline CAPS Events & 4.81 & 2.23 & 6.60 & 2.19 & 5.00 & .03 \\
\hline Checklist & 7.75 & 4.96 & 14.80 & 5.63 & 10.81 & .001 \\
\hline CAPS-I & 7.25 & 5.62 & 22.70 & 6.14 & 22.60 & $<.0001$ \\
\hline CAPS-A & 3.50 & 5.28 & 26.10 & 6.10 & 26.05 & $<.0001$ \\
\hline CAPS-H & 2.94 & 4.55 & 20.10 & 5.99 & 24.06 & $<.0001$ \\
\hline CAPS Sum & 13.69 & 12.90 & 68.90 & 15.46 & 25.99 & $<.0001$ \\
\hline
\end{tabular}

Notes. All tests were two-tailed. As none of the nontraumatized control experienced any traumatic event, merely the values of traumatized controls and PTSD patients are depicted. PTSD = posttraumatic stress disorder; CAPS $=$ Clinician Administered PTSD Scale; CAPS Events $=$ sum CAPS event list; Checklist $=$ vivo Checklist of Organized Violence; CAPS $\mathrm{I}=\mathrm{CAPS}$ intrusion subscale; CAPS-A =CAPS avoidance subscale CAPS-H $=$ CAPS hyperarousal subscale; CAPS Sum = CAPS sum score; $M=$ mean; $S D=$ standard deviation.

non-PTSD subjects, and 11 healthy controls who never experienced any traumatic stressor. In three traumatized controls, an earlier PTSD had remitted. The investigation was split: at the first meeting, the purpose and the course of the investigation were explained in detail, informed consent was acquired, and diagnostic procedures took place. MRI measurements were conducted on a separate day at the University Hospital of Magdeburg. Participants received a compensation of 70 EUR. All procedures were in accordance with the Declaration of Helsinki and approved by the Ethics Committee of the University of Konstanz.

This study population has, in a slightly different composition, already been described elsewhere (Eckart et al., 2011). Participants' mean age was 36.1 years in PTSD subjects $(S D=7.7,23-55$ years), 34.1 years in traumatized controls $(S D=10.1,21-53$ years) and 30.2 years in controls $(S D=7.0,22-48$ years). Groups did not differ significantly in age. Subjects were mainly Kurdish $(n=43)$. The remaining participants were Albanian $(n=1)$, Serbian $(n=1)$, Romani $(n=1)$, and Turkish $(n=1)$. Forty-five participants were right handed and two subjects (one control and one PTSD subject) were left handed according to the Edinburgh Handedness Inventory (Oldfield, 1971). One subject (of the PTSD group) occasionally had taken antidepressant medication (as a hypnotic). Other than that, none of the subjects consumed any psychoactive drug or medication.

Most of the traumatized participants had been repeatedly exposed to a variety of severe traumatic stressors. Subjects were between 5 and 32 years of age when they experienced their first traumatic event (mean age 15.5, SD=5.9). The two traumatized groups did not differ regarding their age at first traumatic experience. PTSD subjects reported a greater number of different types of traumatic events (see Table 1 for mean values and standard deviations of clinical instruments). Groups differed, as a trend, in the number of adverse childhood experiences as measured with the Childhood Trauma Questionnaire (CTQ; controls: $M=37.18$, $S D=4.83$; traumatized controls: $M=38.06, S D=5.34$; PTSD patients: $M=42.65, S D=8.74$; Kruskal-Wallis $\chi^{2}(2)=5.64$, $p=.06)$. Post hoc comparisons revealed a trend for a difference between controls and PTSD patients $(p=.10)$ but not between the other groups. Seventeen participants (one in each control group and 15 in the PTSD group) fulfilled criteria for major depression according to DSM-IV (American Psychiatric Association, 1994). 


\section{Diagnostic Interviews and Memory Test}

Interviews were structured and administered in the mother tongue of the participants with the aid of trained interpreters. Initially, sociodemographic information was obtained. Subsequently, diagnostic procedures proceeded as follows.

Adverse childhood experiences were evaluated with the CTQ (Bernstein et al., 1994), a retrospective, self-report inventory to examine emotional, physical/sexual abuse and neglect during childhood (possible range: $34-170$ ). Exposure to traumatic stressors was quantified with a shortened version of the Vivo Checklist of War, Detention and Torture Events (Schauer, Neuner, \& Elbert, 2011), a scale consisting of 28 imprisonment- and war-related traumatic event types (e.g., being beaten, receiving electrical shocks, or experiencing bombings) (possible range: 0-28). Current and lifetime PTSD symptoms were assessed with the Clinician Administered PTSD Scale (CAPS; Blake et al., 1995), a 30-item, structured interview corresponding to PTSD criteria according to DSM-IV (American Psychiatric Association, 1994) (possible range: 0-136). Diagnosis of major depression, suicidal ideations, and alcohol or substance dependency or abuse according to DSM-IV was based on the corresponding sections of the MiniInternational Neuropsychiatric Interview (M.I.N.I.; Sheehan et al., 1998).

Participants were tested with a nonverbal, culture-independent memory test for the places of objects (MP test; Elbert et al., 2009). Thereto, up to ten small, familiar objects (e.g., a toy car, a spool, a ball) were placed in front of the subject for a memorization phase of $30 \mathrm{~s}$. Afterwards objects were hidden under opaque cups for a delay period of $2 \mathrm{~min}$. The identical objects and three distracter items were then handed to the participant, who had to put each object on top of the respective cup where its counterpart was hidden. The test was conducted in three stages of increasing difficulty with five, seven, and ten hidden items. The number of incorrectly assigned objects was counted. This test has already been used to test memory functions of traumatized children and proved to be sensitive for PTSD-related memory disturbances (Elbert et al., 2009).

\section{MRI Acquisition and Data Analyses}

MRI acquisition. High resolution, T1-weighted structural MRI scans of the brain were acquired on a 3 T Siemens MAGNETOM Trio scanner (Siemens, Erlangen, Germany) with an 8-channel phased-array head coil using a 3D-MPRAGE sequence (TE [echo time] $=4.77 \mathrm{~ms}$, TR [repetition time] $=2500 \mathrm{~ms}$, TI [inversion time $]=1100 \mathrm{~ms}, \quad$ flip angle $=7^{\circ}, \quad$ bandwidth $=140 \mathrm{~Hz} /$ pixel, matrix $=256 \times 256 \times 192$, isometric voxel size $=1.0 \mathrm{~mm}^{3}$ ).

Manual volumetry of the hippocampus. The volume of the hippocampus was determined manually by a rater extensively trained in hippocampal anatomy, who was blind to all clinical and demographic information. Gray matter voxels belonging to the hippocampus were labeled on $\mathrm{T} 1$-weighted images in all three dimensions with MRIcron software (www.mricro/mricron), following standardized guidelines (Pruessner et al., 2000). As we aimed to include exclusively gray matter voxels, alveus, fimbria, and the dentate fissure were omitted. Eight subjects were assessed twice by the main rater and by an independent second rater. Intraclass correlation coefficients (ICC) were calculated. Intrarater ICCs were .96 for the left and .97 for the right hippocampus. Interrater ICCs were .83 for the left and .91 for the right hippocampus.
Volume determination of the insula. To determine insular volumes, the cortical reconstruction and volumetric segmentation procedure offered by FreeSurfer (http://surfer.nmr.mgh.harvard. edu/) was implemented. The technical details of these procedures are described elsewhere (Dale, Fischl, \& Sereno, 1999; Dale \& Sereno, 1993; Fischl, Sereno, \& Dale, 1999). In short, each scan is registered into Talairach space, intensity corrected, and skullstripped (Segonne et al., 2004). Images are then segmented to identify the boundary between gray and white matter and to create a surface representation of the cortex (Dale et al., 1999; Dale \& Sereno, 1993; Fischl et al., 1999). Finally, the cortex is parcellated into units based on its gyral and sulcal structure, and volumes for each section are calculated. This method proved to be fairly accurate with a median accuracy of $80 \%$ in the left and $79 \%$ in the right hemisphere (Fischl et al., 2004). The insula was defined as the sum of the central sulcus of the insula, the short and long gyrus of the insula, and the anterior, inferior, and superior part of the circular sulcus of the insula.

MRS data acquisition and analysis. Single voxel ' $\mathrm{H}$ MR spectra (PRESS, TE $=135 \mathrm{~ms}, \mathrm{TR}=2000 \mathrm{~ms}, 256$ averages, bandwidth $=1200 \mathrm{~Hz}$, acquisition time $=853 \mathrm{~ms}$ ) of bilateral hippocampi and bilateral insulae were recorded subsequent to the high resolution T1-weighted scan. Voxels comprising the medial and posterior part of the hippocampus (voxel size $=2 \times 1 \times 1 \mathrm{~cm}^{3}$ ) or the insula (voxel size $=3 \times 1 \times 1.5 \mathrm{~cm}^{3}$ ) were individually positioned in the subjects' brains. Generally, manual shimming was performed to improve magnetic field homogeneity set by the automatic shim routine. Additionally, water reference data with radiofrequency pulses for water suppression switched off (TR $=10 \mathrm{~s}, 4$ averages) were acquired for eddy current correction and scaling of the metabolite concentrations to the internal water content. Spectra were analyzed using LCModel version 6.1.0 (www.s-provencher. com/pages/lcmodel.shtml). Spectra with full-width-half-maximum line widths larger than $10 \mathrm{~Hz}$ and quantification results with a Cramér-Rao lower bound higher than $12 \%$ were excluded from further analysis. Ten measurements (seven in the right and three in the left hippocampus) were discarded due to these reasons.

Water reference scans were corrected for (a) voxel volume fractions of gray matter, white matter, and cerebrospinal fluid (CSF) extracted from SPM5-based tissue segmentation (Wellcome Department of Cognitive Neurology, Institute of Neurology, London, UK), (b) tissue water content using standard values published by Ernst and colleagues (Ernst, Kreis, \& Ross, 1993), and (c) water relaxation times either published (Rooney et al., 2007; Stanisz et al., 2005) or drawn from our lab data base. Similarly, relaxation times for NAA and creatine measured in the hippocampus and in pure white matter from our laboratory data base were used to correct metabolite concentrations. Metabolite concentrations are given in mmol per liter tissue.

To determine the percentage of tissue within each MRS voxel that could unambiguously be identified as hippocampal tissue, the individual manual segmentations of the hippocampus and the respective MRS voxels were coregistered. On average, 25\% $(S D=6.1)$ of the left hippocampal MRS voxel and $24.2 \%$ $(S D=5.9)$ of the right hippocampal MRS voxel contained unambiguous hippocampal tissue.

\section{Statistical Analysis}

Population characteristics. Population characteristics and clinical parameters were compared using analyses of variance 
(ANOVAs) and Kruskal-Wallis rank sum tests. For post hoc comparisons, pairwise $t$ tests and pairwise Wilcoxon rank sum tests were used. All post hoc tests were corrected for multiple comparisons according to Hommel (Hommel, 1989). Count data was analyzed using Fisher's exact tests.

Brain measures. Volumetric and spectroscopic measures were compared with linear mixed-effects models including hemisphere as within-subjects factor (Pinheiro \& Bates, 2000). Volumetric analyses were adjusted for intracranial volume and age by including these variables in the statistical models as covariates of no interest. Spectroscopic analyses were adjusted for age as well as for the fraction of gray and white matter within the MRS voxel. If a significant Hemisphere $\times$ Group interaction indicated a laterality effect, analyses were repeated separately for each hemisphere. Associations between clinical parameters/memory performance and brain measures were initially explored using Pearson's product-moment correlations. If a significant association was revealed, the variable was included in the primary linear model. A variable was interpreted as being influential if this model was favored by a corresponding likelihood ratio test. In an attempt to identify a potential influence of depression symptoms on our results, we initially included this variable in our statistical models - however, as no significant influence of depression symptoms has been revealed, this parameter was excluded from the final models.

MP test. Group differences in general memory performance were investigated with a linear mixed-effects model including the stages of the MP test as within-subjects factor. To clarify at which level and between which groups the number of errors differed, post hoc tests, corrected for multiple comparisons, were calculated for each stage of the MP test.

All analyses were conducted using the statistical program $R(R$ Development Core Team, 2007; version 2.7.1) with the additional package NLME (Pinheiro, Bates, DebRoy, Sarkar, \& R Core Team, 2008; version 3.1-90).
Results

\section{Volumetry}

Groups neither differed in hippocampal, $F(2,41)=.06, p=.94$, nor in insular volumes, $F(2,42)=.85, p=.43$ (see Figure 1 for a graphic depiction of raw values of bilateral hippocampus and insula volumes). No association between volumetric measures and traumatization and/or PTSD symptoms was revealed. See online Supporting Information for mean volumes and standard deviations of bilateral hippocampus and insula. Inclusion of depression as a covariate did not alter these results.

\section{MR Spectroscopy}

No significant group difference was found in hippocampal NAA concentrations, $F(2,42)=1.16, p=.32$, or NAA/creatine ratios, $F(2,42)=.38, p=.69$. Groups did not differ in hippocampal creatine concentrations, $F(2,42)=2.18, p=.13$. The proportional fraction of factual hippocampal tissue was $25.0 \%$ for the left and $24.2 \%$ for the right hippocampus. Groups did not differ in insular NAA concentrations, $F(2,43)=.51, p=.61$, or NAA/creatine ratios, $F(2,43)=.61, p=.55$. Groups did not differ in insular creatine concentrations, $F(2,43)=.76, p=.48$ (see Figure 2 for a graphic depiction of mean values and standard errors of NAA and NAA/ creatine levels in bilateral hippocampus and insula). There was no association between spectroscopic measures and traumatization and/or PTSD symptoms. See on-line Supporting Information for mean metabolite concentrations and standard deviations in bilateral hippocampus and insula. Inclusion of depression as a covariate did not alter these results.

\section{The Childhood Trauma Questionnaire}

A relation between the sum score of the CTQ and NAA levels in the left hippocampus was revealed. The CTQ was significantly associated with the NAA level, $t(43)=-2.57, p=.01$, and with the NAA/creatine ratio as a trend, $t(43)=-1.67, p=.10$. Whereas the
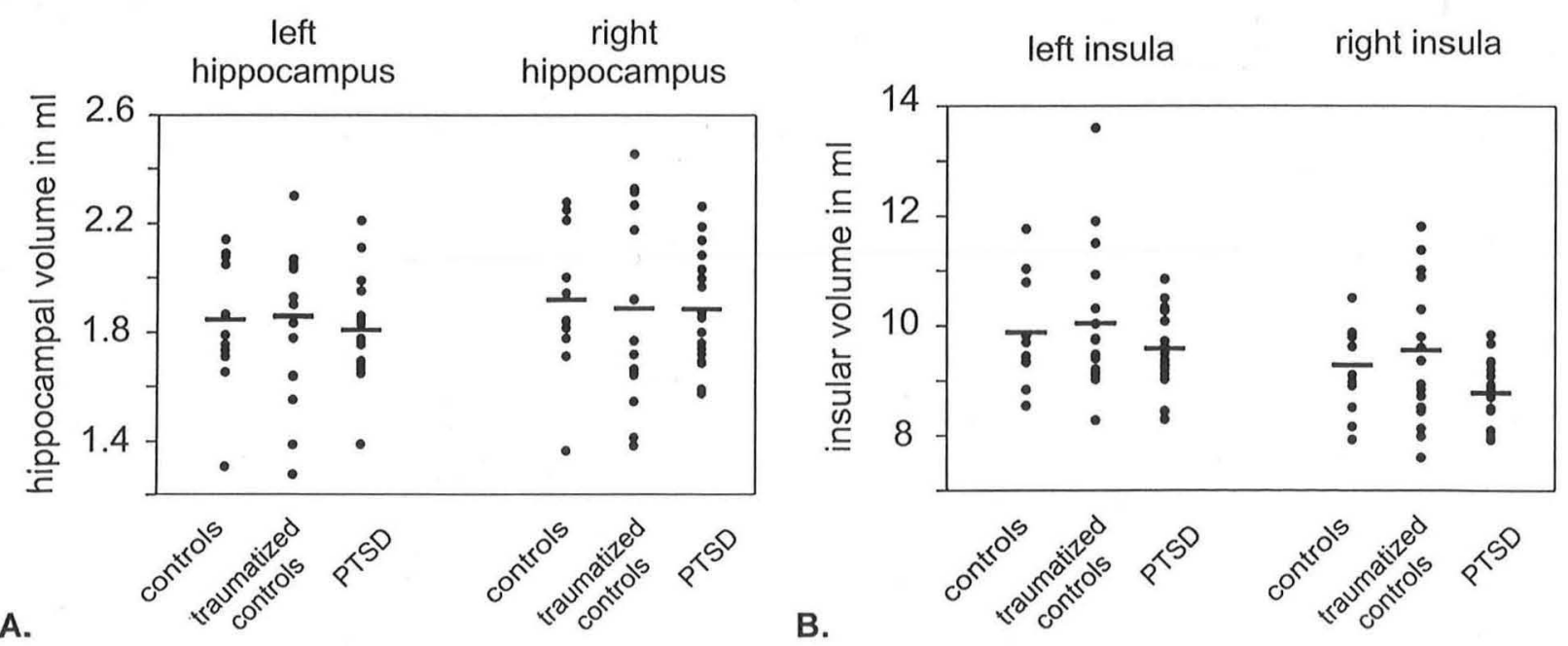

Figure 1. Volumetry. Graphic depiction of the raw values of bilateral hippocampus (A) and insula (B) volumes of participants subdivided into groups. Horizontal bars indicate mean volumes within the groups. No significant group difference was revealed in hippocampal volumes, also when corrected for age and intracranial volume. Precise statistical parameters are presented within the main text. PTSD = post-traumatic stress disorder. 

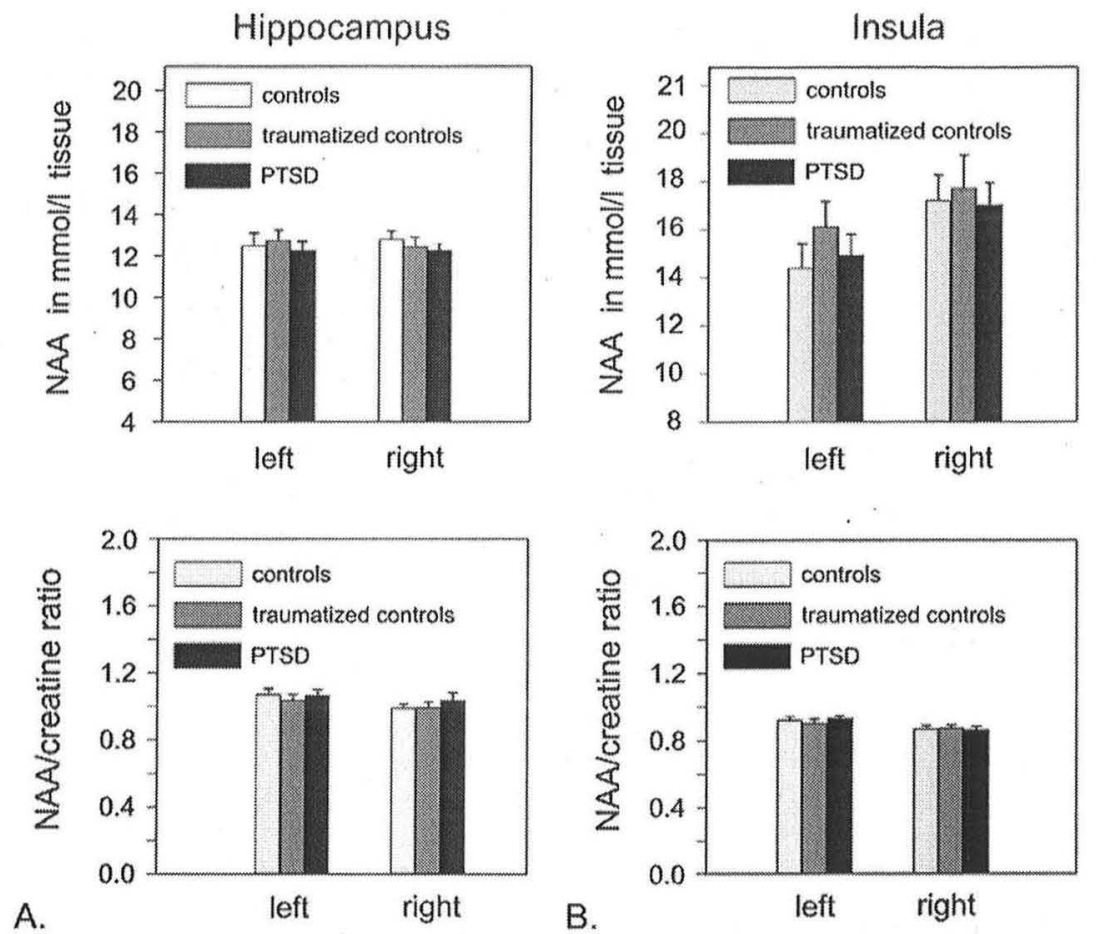

Figure 2. Spectroscopy. Graphic depiction of means and standard errors of hippocampal NAA concentration and the respective NAA/creatine ratios in hippocampus (A) and insula (B). No significant group differences were revealed in hippocampal NAA levels or respective NAA/creatine ratios. Precise statistical parameters are presented within the main text. PTSD $=$ post-traumatic stress disorder.

model with the NAA concentration was favored by the likelihood ratio test, $\chi^{2}(4)=6.58, p=.04$, the model with the NAA/creatine ratio was rejected, $\chi^{2}(4)=2.89, p=.23$. As only a minority of subjects reported adverse childhood experiences, variance of the CTQ might have been distorted. Thus, a subgroup of participants (independent of diagnostic group) was formed that actually had experienced adverse childhood events (all subjects scoring higher than or equal to three ("sometimes true") in at least two items of the CTQ, $n=16$, ten PTSD patients, four traumatized controls, two nontraumatized controls, $M=47.88, S D=7.16$ ). Analyses were repeated for this subpopulation: again, the CTQ was strongly associated with the NAA level, $t(43)=-3.54, p=.004$, and the NAA/ creatine ratio, $t(12)=-3.55, p=.004$ the higher the CTQ score, the lower the metabolite level in the left hippocampus (see Figure 3 for a graphic depiction of the association between the CTQ and NAA levels in this subsample of subjects). Likelihood ratio tests favored the models including the NAA level, $\chi^{2}(4)=10.72$, $p=.005$, and the NAA/creatine ratio, $\chi^{2}(4)=10.78, p=.005$. No corresponding associations between negative childhood experiences and hippocampal volume data were revealed. Inclusion of depression as a covariate did not alter these results.

\section{Memory Test}

Subjects with PTSD performed poorer in the MP test than both control groups. A repeated measures ANOVA (including the course of the memory test as within-subjects factor) revealed more errors in the PTSD group than in controls, $F(2,44)=5.62, p=.007$ (controls vs. PTSD: $t(44)=2.55, p=.01$; traumatized controls vs. PTSD: $t(44)=3.01, p=.004$; controls vs. traumatized controls: $t(44)=-.14, p=.89)$. The poorer performance of PTSD subjects was prominent at testing with seven items, $F(2,44)=3.46, p=.04$ (controls vs. PTSD: $p=.09$, traumatized controls vs. PTSD: $p=.06$, controls vs. traumatized controls: $p=.97$ ) and ten items, $F(2,44)=4.00, p=.03$ (controls vs. PTSD: $p=.09$, traumatized controls vs. PTSD: $p=.04$, controls vs. traumatized controls: $p=.83$ ) but not five items, $F(2,44)=.96, p=.39$ (see Figure 4 for mean number of errors and standard errors at different time points). Memory performance was associated with the NAA level, $t(42)=-2.25, p=.03$, of the left hippocampus, irrespective of group membership. The lower the neurometabolite level the poorer the subject's performance in the MP test. A likelihood ratio test favored the model including the NAA concentration, $\chi^{2}(1)=14.97$, $p<.0006$. The interaction between group membership and NAA levels failed to reach significance, $F(2,40)=2.29, p=.11$. However, as the groups differed in their performance in the MP test, we considered the association separately for each group. The relationship appears to be driven by the PTSD group, $t(17)=-2.46$, $p=.02$, but not by the other two groups $(p>60)$. Inclusion of depression as a covariate did not alter these results.

\section{Discussion}

We combined MR spectroscopy and volumetric analyses to investigate the influence of traumatic stress experiences and PTSD on hippocampus and insula of highly traumatized refugees. By choosing a population without a history of alcohol/substance abuse or other comorbid psychiatric conditions and/or regular psychiatric medication, we controlled for confounding variables that frequently have hampered PTSD-related brain research. Indeed, a 


\section{Left Hippocampus}
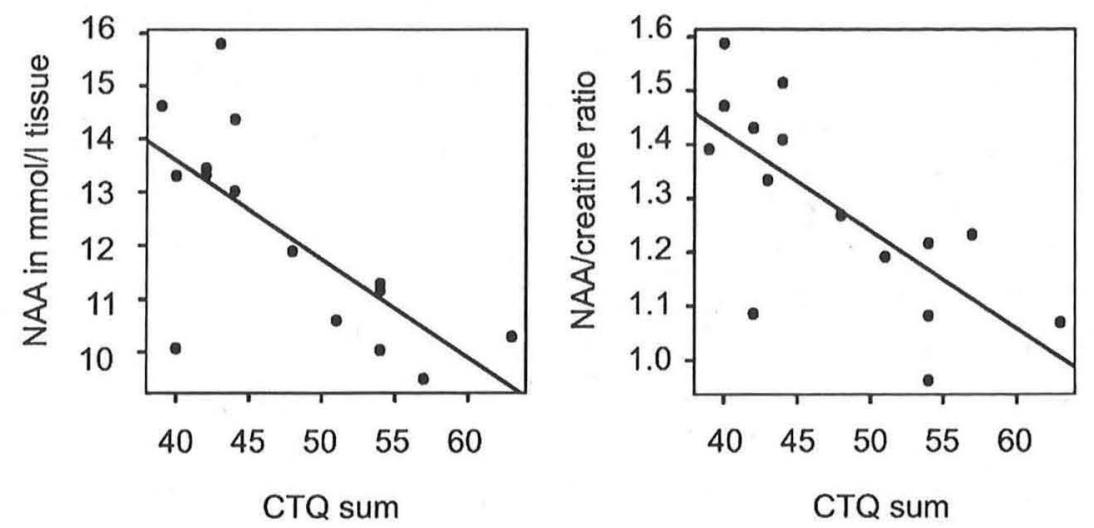

Figure 3. Graphic depiction of an association between negative childhood experiences (quantified with the CTQ) and the NAA concentration in the left hippocampus. Depicted are raw values. As only a minority of subjects reported adverse childhood experiences, a subgroup of participants was formed that actually had experienced adverse childhood events. The CTQ was strongly associated with left hippocampal NAA and the NAA/creatine ratio in this sample-the higher the CTQ score, the lower the metabolite level in the left hippocampus. CTQ=Childhood Trauma Questionnaire; $\mathrm{NAA}=\mathrm{N}$-acetyl-aspartate.

generally poorer performance of PTSD subjects in a test of spatial memory indicated functional impairments that may be attributed to the hippocampus. However, no group differences were revealed in volumes or NAA concentrations of bilateral hippocampus and insula, even though an association between left hippocampal NAA and adverse childhood experiences (irrespective of PTSD diagnosis) indicated a detrimental effect of these events on hippocampal integrity. The potential role of the insula in the pathophysiology of

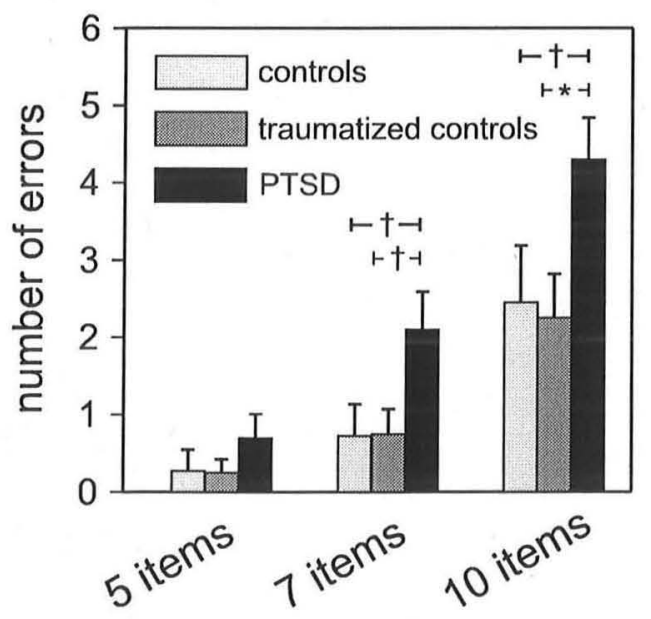

Figure 4. Graphic depiction of group differences in different levels of the MP test. Depicted are mean number of errors and standard errors. Groups significantly differed in the overall performance in the MP test, with PTSD patients showing a poorer performance than both control groups. After correction for multiple comparisons, groups differed (at least as a trend) at testing with seven and ten items. Precise statistic parameters are presented within the main text. PTSD $=$ posttraumatic stress disorder; $\uparrow=$ indicating a trend for a group difference after correction for multiple comparisons $(p \leq .10) ; *=$ indicating a significant group difference after correction for multiple comparisons $(p \leq .05)$.
PTSD has just recently been highlighted (Liberzon \& Martis, 2006). Strong support for this notion came from a plethora of neuroimaging studies documenting altered insular activity in PTSD (Etkin \& Wager, 2007; Kolassa et al., 2007; Liberzon \& Martis, 2006). However, as no evidence for insular atrophies was revealed in our sample, these functional impairments might not be accompanied by corresponding macroscopic structural alterations. Accordingly, subsequent discussion will focus on hippocampus data. There might be several reasons why we did not reveal structural alterations in the hippocampus of this specific sample of highly traumatized PTSD patients.

\section{Comorbid Alcohol Abuse}

The hippocampus is one of the core structures within the neurobiological model of PTSD (Elbert \& Schauer, 2002; Kolassa \& Elbert, 2007; Schauer et al., 2011), and reports of alterations in this part of the brain are numerous (Bonne et al., 2008; Bremner et al., 2003; Geuze, Vermetten, \& Bremner, 2005b; Gilbertson et al., 2002; Gurvits et al., 1996; Lindauer et al., 2004; Mohanakrishnan Menon et al., 2003; Schuff et al., 2001, 2008; Shin et al., 2004; Vythilingam et al., 2005). Accordingly, meta-analyses concluded that PTSD patients indeed show reduced hippocampal volumes (Karl et al., 2006) and neurometabolite levels (Karl \& Werner, 2009). However, there have been concerns that the particularly high incidence of comorbid alcohol and/or substance abuse might have distorted previous conclusions (Woodward et al., 2006). Most studies reporting reduced hippocampal volumes (Bremner et al., 2003; Gilbertson et al., 2002; Gurvits et al., 1996; Schmahl et al., 2009; Vythilingam et al., 2005) or NAA levels (Freeman et al., 1998; Mohanakrishnan Menon et al., 2003; Schuff et al., 2001) in PTSD patients include individuals with a history of former alcohol and/or substance abuse. Studies that have, on the other hand, rigorously excluded subjects with a history of alcohol and/or substance abuse did, in the majority of cases, not reveal a PTSD-related reduction in hippocampal volumes (De Bellis et al., 2001; Freeman et al., 2006; Golier 
et al., 2005; Jatzko et al., 2006; Woodward et al., 2006) or NAA levels (Freeman et al., 2006). The additional diagnosis of PTSD, furthermore, did not add to the effects of alcohol consumption on the hippocampal tissue of alcoholic women (Agartz et al., 1999) We explicitly chose a study sample without a history of alcohol/ substance abuse and can thus rule out potential atrophic processes due to repeated alcohol intoxication secondary to PTSD. However, the symptom severity of our subjects was comparable to studies in which PTSD-related hippocampal atrophies were reported (Bonne et al., 2008; Gilbertson et al., 2002; Gurvits et al., 1996; Shin et al., 2004; Woodward et al., 2006), irrespective of the occurrence of comorbid alcohol abuse within those populations (Gilbertson et al., 2002; Gurvits et al., 1996; Woodward et al., 2006). This precludes the possibility that a generally lower symptom load in our sample might have entailed both a lower rate of psychiatric comorbidities and a lack of brain structural alterations. Accordingly, our finding of no PTSD-related differences in hippocampal volumes or NAA levels might be interpreted as support for the notion that at least part of the hippocampal alterations reported in the literature could be associated with comorbid alcohol and/or substance abuse.

\section{Adverse Childhood Experiences}

Biological mechanisms of brain maturation could be another factor that interacts with the negative effects of traumatic stress on brain structures. Stressful experiences in sensitive developmental stages were suggested to shape the brain to adapt the individual for the prospect of high levels of lifelong stress (Teicher et al. 2003). Indeed, survivors of childhood sexual abuse showed hippocampal volume reductions only if these adverse experiences took place during discernible developmental phases (Andersen et al., 2008). These potential consequences of adverse childhood experiences do not depend on their extreme forms (severe physical or sexual abuse), as the experience of parental verbal aggression (Teicher et al., 2003) and neglect (Sar, Tutkun, Alyanak, Bakim, \& Baral, 2000) have been associated with a higher incidence of psychiatric symptoms and even with structural brain alterations (Choi, Jeong, Rohan, Polcari, \& Teicher, 2009) as well A large number of studies reporting PTSD-related alterations in the hippocampus investigated survivors of childhood abuse (Bremner et al., 2003; Schmahl et al., 2009) or war veterans (Freeman et al., 1998; Gilbertson et al., 2002; Gurvits et al., 1996; Mohanakrishnan Menon et al., 2003; Schuff et al., 2001, 2008; Vythilingam et al., 2005). In the latter population, a high occurrence of familial instability, neglect, or physical/sexual abuse and its influence on later development of PTSD has repeatedly been reported (Gahm, Lucenko, Retzlaff, \& Fukuda, 2007; Zaidi \& Foy, 1994). Thus, the occurrence of hippocampal alterations in these populations might, at least partly, be attributable to a relatively high load of adverse childhood experiences. In line with this notion, our population, in which the incidence of detrimental events during childhood was generally rather low, showed no group differences in hippocampal volumes or neurometabolites. Moreover, the only psychological measure that showed an association with hippocampal measures was the CTQ - indicating that negative childhood experiences might indeed have a negative effect on hippocampal integrity. The occurrence of these events (traumatic and/or nontraumatic) might thus be crucial for the manifestation of hippocampal atrophies and/or render the individual vulnerable for the biological and psychological consequences of later traumatic stress.

\section{Memory Test}

Even though no macroscopically detectable reductions in the volume or NAA levels in the hippocampus were revealed, PTSD patients indeed showed a poorer performance in the MP test-a test that has already been shown to be sensitive for PTSD-related memory impairments (Elbert et al., 2009). PTSD has frequently been linked to specific cognitive abnormalities (McNally, 2006), and an impairment of hippocampal functioning has been reported independently of structural brain atrophies (Bremner et al., 2003; Golier et al., 2005). However, even though an association between PTSD and impaired verbal abilities is well documented (Johnsen \& Asbjornsen, 2008), it has recently been questioned that declarative memory functions should inevitably be disturbed in PTSD patients (Woodward et al., 2009). The MP test relies on the memorization of the places of objects, a function that has (at least in the animal) been linked to the hippocampus (Rolls, Xiang, \& Franco, 2005). The PTSD patients of our sample were indeed impaired in this test, and their performance seemed related to reduced NAA levels of the left hippocampus. These results indicate that PTSD patients show memory deficits (that might, however, be very specific) and suggest these disturbances may be linked to hippocampal functions. However, it has to be considered in this context that the MP test is not psychometrically characterized so far, and that its results should thus be interpreted with caution.

\section{Methodological Aspects}

In particular for our spectroscopic results, the discrepancy between this study and reports of PTSD-related reductions in neurometabolite concentrations (Freeman et al., 1998; Mohanakrishnan Menon et al., 2003; Schuff et al., 2001, 2008) might as well rely on methodological differences between the studies. Previous single-voxel spectroscopy investigations used rather large voxel sizes (ranging from $4 \mathrm{~cm}^{3}$ (Freeman et al., 2006) to more than $9 \mathrm{~cm}^{3}$ (Freeman et al., 1998; Mohanakrishnan Menon et al., 2003)), or cubic voxels (Mohanakrishnan Menon et al., 2003), which might, however, not be well suited to the elongated anatomy of the hippocampus. Accordingly, these analyses might have been particularly susceptible for partial volume effects. Our voxels were substantially smaller. Still, the fraction of hippocampal gray matter within these voxels was relatively low. Reports of reduced metabolite concentrations in larger voxels (Freeman et al., 1998; Mohanakrishnan Menon et al., 2003) might thus be attributable to general alterations in the medial temporal lobe rather than being specific to the hippocampus. Furthermore, the MRS voxels were positioned in the anterior part of the hippocampus in most previous investigations (Freeman et al., 1998; Mohanakrishnan Menon et al., 2003). According to our experience, this region, however, cannot be shimmed with consistently high quality due to high interindividual anatomic differences. Thus, our decision to place the voxel in the more medial part of the hippocampus further enhanced the quality of spectra. Finally, MRS quantification results are not only biased by the line width of and the signal-to-noise ratio within the spectra, but also by the quantification approach itself (Kanowski, Kaufmann, Braun, Bernarding, \& Tempelmann, 2004). Accordingly, the variety of quantification software implemented in MRS studies on PTSD (Mohanakrishnan Menon et al., 2003; Schuff et al., 2001) might further account for some discrepant findings. 


\section{Limitations}

Some methodological issues should be considered when interpreting the present results. Comparable to previous studies (e.g., Woodward et al., 2009), 75\% of our PTSD patients suffered from comorbid depression. Including depression as covariate of no interest did not alter our results, Moreover, it has generally been suggested that major depression and PTSD symptoms might emerge simultaneously within the posttraumatic psychopathology (Kasai et al., 2008; O'Donnell et al., 2004). A division between these conditions would thus be artificial and might not adequately reflect the clinical reality in chronic PTSD (Miller \& Chapman, 2001). However, as we did not include a "depression-only" group, we cannot fully distinguish between the effects of these psychiatric conditions on our results. The high comorbidity with major depression should thus be kept in mind, and conclusions about the PTSD-specificity of our results should be drawn with caution. Another population-specific concern is intrinsically linked to the choice of our sample. Even though the investigation of a refugee population enabled us to circumvent many methodological confounds that have been discussed in PTSD-related brain research (e.g., high comorbid alcohol consumption, heterogeneous trauma history), this sample substantially differed from previous samples in cultural background. Thus, we cannot explicitly preclude that some unidentified cultural resilience factors (e.g., perceived social support, religious background) might have influenced our results.

Another line of concern affects methodological issues. As previous MRS investigations faced substantial problems regarding partial volume effects (Freeman et al., 1998, 2006), we have deliberately chosen relatively small voxel sizes. However, as mentioned above, just a small fraction of gray matter within our voxels could unambiguously be identified as hippocampal gray matter. The spectroscopic signal arising from this fraction could accordingly not have been strong enough to reveal hippocampal atrophy, even if it would have been present. However, we tried to account for this issue by including the fraction of hippocampal tissue within the voxels as a covariate in our statistical models. This procedure did not alter the results. We specifically tailored our MRS protocol to circumvent some methodological problems (e.g., voxel sizes and location, metabolite quantification) of previous MRS studies in PTSD patients. Even though this procedure militates in favor of the quality of our MRS spectra, it might have been at the expense of the comparability of our work with previous studies, and corresponding comparisons should be drawn with caution. However, general support for the validity of our hippocampal NAA signals might come from our correlational analyses-we replicated previous reports of an association between negative childhood experiences and hippocampal atrophy (Andersen et al., 2008), as well as medial temporal lobe NAA levels and memory performance (Giménez et al., 2004), what militates in favor of the general validity of the hippocampal NAA levels detected in our subjects. Finally, even though our sample sizes (20 PTSD patients vs. 16 traumatized controls vs. 11 nontraumatized controls) were comparable to or even higher than sample sizes in studies that indeed reported PTSD-related hippocampal atrophies (e.g., Bremner et al., 2003; Gurvits et al., 1996; Lindauer et al., 2004; Schmahl et al., 2009; Shin et al., 2004), groups were still rather small for the statistical approaches chosen. Accordingly, we cannot exclude the possibility that the lack of group differences in the analyzed brain areas might be due to low statistical power.

\section{Conclusions and Future Perspectives}

The present results may add to some existing evidence that the experience of traumatic stress and/or the development of PTSD symptoms are not necessarily tied to pronounced hippocampal atrophy. Rather, PTSD-related alterations in this structure might in some cases be a consequence of early adversity or result from other factors, such as the extensive use of psychoactive drugs, including alcohol. Moreover, it has to be emphasized that disturbed brain functions are not inevitably attended by corresponding macroscopic structural alterations but can, as our insula findings suggest, certainly occur without them. By investigating the insula and the hippocampus of highly traumatized refugees, we deliberately chose core regions in the neurobiological model of PTSD. Previous work in the same sample, furthermore, showed that PTSD-related structural alterations were present in frontal brain regions as well (Eckart et al., 2011). Accordingly, the neuronal network implicated in PTSD seems to be widespread, and symptom development might be the result of a complex interplay between functional and structural disturbances within this network. Future research should be particularly concerned with the identification and understanding of brain regions that are involved in the emergence of the disease. In doing so, the contribution of other structures (e.g., the anterior cingulate cortex; Ham et al., 2007; Kasai et al., 2008; Liberzon \& Martis, 2006) that proved to be implicated in PTSD should be given special attention.

\section{References}

Agartz, I., Momenan, R., Rawlings, R. R., Kerich, M. J., \& Hommer, D. W. (1999). Hippocampal volume in patients with alcohol dependence. Archives of General Psychiatry, 56, 356-363. doi: 10.1001/ archpsyc.56.4.356

Alvarez, R. P., Biggs, A., Chen, G., Pine, D. S., \& Grillon, C. (2008). Contextual fear conditioning in humans: Cortical-hippocampal and amygdala contributions. Journal of Neuroscience, 28, 6211-6219. doi: 10.1523/JNEUROSCI.1246-08.2008

American Psychiatric Association. (1994). Diagnostic and statistical manual of mental disorders (4th ed.). Washington, DC: American Psychiatric Association.

Andersen, S. L., Tomada, A., Vincow, E. S., Valente, E., Polcari, A., \& Teicher, M. H. (2008). Preliminary evidence for sensitive periods in the effect of childhood sexual abuse on regional brain development. Journal of Neuropsychiatry and Clinical Neurosciences, 20, 292-301. doi: 10.1176/appi.neuropsych.20.3.292
Augustine, J. R. (1996). Circuitry and functional aspects of the insular lobe in primates including humans. Brain Research. Brain Research Reviews, 22, 229-244. doi: 10.1016/S0165-0173(96)00011-2

Bernstein, D. P., Fink, L., Handelsman, L., Foote, J., Lovejoy, M., \& Wenzel, K., ... Ruggiero, J. (1994). Initial reliability and validity of a new retrospective measure of child abuse and neglect. American Journal of Psychiatry, 151, 1132-1136.

Blake, D. D., Weathers, F. W., Nagy, L. M., Kaloupek, D. G., Gusman, F. D., \& Charney, D. S., \& Keane, T. M. (1995). The development of a clinician-administered PTSD scale. Journal of Traumatic Stress, 8, 75-90. doi: 10.1002/jts.2490080106

Bonne, O., Brandes, D., Gilboa, A., Gomori, J. M., Shenton, M. E., \& Pitman, R. K., \& Shalev, A. Y. (2001). Longitudinal MRI study of hippocampal volume in trauma survivors with PTSD. American Journal of Psychiatry, 158, 1248-1251. doi: 10.1176/appi.ajp. 158.8 .1248 
Bonne, O., Vythilingam, M., Inagaki, M., Wood, S., Neumeister, A., Nugent, A. C., ... Charney, D. S. (2008). Reduced posterior hippocampal volume in posttraumatic stress disorder. Journal of Clinical Psychiatry, 69, 1087-1091. doi: 10.4088/JCP.v69n0707

Bremner, J. D., Vythilingam, M., Vermetten, E., Southwick, S. M., McGlashan, T., Nazeer, A., \& Charney, D. S. (2003). MRI and PET study of deficits in hippocampal structure and function in women with childhood sexual abuse and posttraumatic stress disorder. American Journal of Psychiatry, 160, 924-932. doi: 10.1176/appi.ajp.160.5.924

Choi, J., Jeong, B., Rohan, M. L., Polcari, A. M., \& Teicher, M. H. (2009). Preliminary evidence for white matter tract abnormalities in young adults exposed to parental verbal abuse. Biological Psychiatry, 65, 227-234. doi: 10.1016/j.biopsych.2008.06.022

Craig, A. D. (2002). How do you feel? Interoception: The sense of the physiological condition of the body. Nature Reviews Neuroscience, 3, 655-666. doi: 10.1038/nrn894

Craig, A. D. (2009). How do you feel-now? The anterior insula and human awareness. Nature Reviews Neuroscience, 10, 59-70. doi: $10.1038 / \mathrm{nrn} 2555$

Dale, A. M., Fischl, B., \& Sereno, M. I. (1999). Cortical surface-based analysis. I. Segmentation and surface reconstruction. NeuroImage, 9, 179-194. doi: 10.1006/nimg. 1998.0395

Dale, A. M., \& Sereno, M. I. (1993). Improved localization of cortical activity by combining EEG and MEG with MRI cortical surface reconstruction: A linear approach. Journal of Cognitive Neuroscience, 5, 162-176. doi: 10.1162/jocn.1993.5.2.162

De Bellis, M. D., Hall, J., Boring, A. M., Frustaci, K., \& Moritz, G. (2001). A pilot longitudinal study of hippocampal volumes in pediatric maltreatment-related posttraumatic stress disorder. Biological Psychiatry, 504, 305-309. doi: 10.1016/S0006-3223(01)01105-2

de Quervain, D. J., Kolassa, I. T., Ertl, V., Onyut, P. L., Neuner, F., Elbert, T., \& Papassotiropoulos, A. (2007). A deletion variant of the alpha2badrenoceptor is related to emotional memory in Europeans and Africans. Nature Neuroscience, 10, 1137-1139. doi: 10.1038/nn1945

Driessen, M., Herrmann, J., Stahl, K., Zwaan, M., Meier, S., Hill, A., \& Petersen, D. (2000). Magnetic resonance imaging volumes of the hippocampus and the amygdala in women with borderline personality disorder and early traumatization. Archives of General Psychiatry, 57, 1115-1122. doi: 10.1001/archpsyc.57.12.1115

Eckart, C., Stoppel, C., Kaufmann, J., Tempelmann, C., Hinrichs, H., Elbert, T., \& Kolassa, I.-T. (2011). Structural alterations in lateral prefrontal, parietal and posterior midline regions of men with chronic posttraumatic stress disorder. Journal of Psychiatry and Neuroscience, 36, 176-186. doi: 10.1503/jpn.100010

Elbert, T., \& Schauer, M. (2002). Burnt into memory. Nature, 419, 883. doi: 10.1038/419883a

Elbert, T., Schauer, M., Schauer, E., Huschka, B., Hirth, M., \& Neuner, F (2009). Trauma-related impairment in children-A survey in Sri Lankan provinces affected by armed conflict. Child Abuse \& Neglect. 33, 238-246. doi: 10.1016/j.chiabu.2008.02.008

Ernst, T., Kreis, R., \& Ross, B. D. (1993). Absolute quantitation of water and metabolites in the human brain. Part I: Compartments and water. Journal of Magnetic Resonance, 102, 1-8.

Etkin, A., \& Wager, T. D. (2007). Functional neuroimaging of anxiety: A meta-analysis of emotional processing in PTSD, social anxiety disorder, and specific phobia. American Journal of Psychiatry, 164, 1476-1488 doi: 10.1176/appi.ajp.2007.07030504

Fischl, B., Sereno, M. I., \& Dale, A. M. (1999). Cortical surface-based analysis. II: Inflation, flattening, and a surface-based coordinate system. Neurolmage, 9, 195-207. doi: 10.1006/nimg.1998.0396

Fischl, B., van der Kouwe, A., Destrieux, C., Halgren, E., Segonne, F., Salat, D. H., ... Dale, A. M. (2004). Automatically parcellating the human cerebral cortex. Cerebral Cortex, 14, 11-22. doi: 10.1093/ cercor/bhg087

Freeman, T., Kimbrell, T., Booe, L., Myers, M., Cardwell, D., Lindquist, D. M., \& Komorowski, R. A. (2006). Evidence of resilience: Neuroimaging in former prisoners of war. Psychiatry Research: Neuroimaging, 146, 59-64. doi: 10.1016/j.pscychresns.2005.07.007

Freeman, T. W., Cardwell, D., Karson, C. N., \& Komoroski, R. A. (1998) In vivo proton magnetic resonance spectroscopy of the medial temporal lobes of subjects with combat-related posttraumatic stress disorder. Magnetic Resonance in Medicine, 40, 66-71. doi: 10.1002/mrm. 1910400110

Gahm, G. A., Lucenko, B. A., Retzlaff, P., \& Fukuda, S. (2007). Relative impact of adverse events and screened symptoms of posttraumatic stress disorder and depression among active duty soldiers seeking mental health care. Journal of Clinical Psychology, 63, 199-211. doi: 10.1002/ jclp. 20330

Geuze, E., Vermetten, E., \& Bremner, J. D. (2005a). MR-based in vivo hippocampal volumetrics: 1. Review of methodologies currently employed. Molecular Psychiatry, 10, 147-159. doi: 10.1038/ sj.mp. 4001580

Geuze, E., Vermetten, E., \& Bremner, J. D. (2005b). MR-based in vivo hippocampal volumetrics: 2. Findings in neuropsychiatric disorders. Molecular Psychiatry, 10, 160-184. doi: 10.1038/sj.mp.4001579

Gilbertson, M. W., Shenton, M. E., Ciszewski, A., Kasai, K., Lasko, N. B., Orr, S. P., \& Pitman, R. K. (2002). Smaller hippocampal volume predicts pathologic vulnerability to psychological trauma. Nature Neuroscience, 5, 1242-1247. doi: 10.1038/nn958

Giménez, M., Junqué, C., Narberhaus, A., Caldú, X., Segarra, D., Vendrell, P., ... Mercader, J. M. (2004). Medial temporal MR spectroscopy is related to memory performance in normal adolescent subjects. NeuroReport, 15, 703-707. doi: 10.1097/00001756-200403220-00026

Golier, J. A., Yehuda, R., De Santi, S., Segal, S., Dolan, S., \& de Leon, M. J. (2005). Absence of hippocampal volume differences in survivors of the Nazi Holocaust with and without posttraumatic stress disorder. Psychiatry Research: Neuroimaging, 139, 53-64. doi: 10.1016/j.pscychresns.2005.02.007

Gurvits, T. V., Shenton, M. E., Hokama, H., Ohta, H., Lasko, N. B. Gilbertson, M. W., ... Pitman, R. K. (1996). Magnetic resonance imaging study of hippocampal volume in chronic, combat-related posttraumatic stress disorder. Biological Psychiatry, 40, 1091-1099. doi: 10.1016/S0006-3223(96)00229-6

Ham, B. J., Chey, J., Yoon, S. J., Sung, Y., Jeong, D. U., Ju Kim, S., ... Lyoo, K. (2007). Decreased N-acetyl-aspartate levels in anterior cingulate and hippocampus in subjects with post-traumatic stress disorder: A proton magnetic resonance spectroscopy study. European Journal of Neuroscience, 25, 324-329. doi: 10.1111/j.14609568.2006.05253.x

Hommel, G. (1989). A comparison of two modified Bonferroni procedures Biometrika, 76, 624-625. doi: 10.1093/biomet/76.3.624

Jatzko, A., Rothenhofer, S., Schmitt, A., Gaser, C., Demirakca, T., WeberFahr, W., ... Braus, D. F. (2006). Hippocampal volume in chronic posttraumatic stress disorder (PTSD): MRI study using two different evaluation methods. Journal of Affective Disorders, 94, 121-126. doi: 10.1016/j.jad.2006.03.010

Johnsen, G. E., \& Asbjornsen, A. E. (2008). Consistent impaired verbal memory in PTSD: A meta-analysis. Journal of Affective Disorders, 111, 74-82. doi: $10.1016 /$ j.jad.2008.02.007

Kanowski, M., Kaufmann, J., Braun, J., Bernarding, J., \& Tempelmann, C. (2004). Quantitation of simulated short echo time $1 \mathrm{H}$ human brain spectra by LCModel and AMARES. Magnetic Resonance in Medicine, 51, 904-912. doi: 10.1002/mrm.20063

Karl, A., Schaefer, M., Malta, L. S., Dorfel, D., Rohleder, N., \& Werner, A. (2006). A meta-analysis of structural brain abnormalities in PTSD. Neuroscience \& Biobehavioral Reviews, 30, 1004-1031. doi: 10.1016/ j.neubiorev.2006.03.004

Karl, A., \& Werner, A. (2009). The use of proton magnetic resonance spectroscopy in PTSD research-Meta-analyses of findings and methodological review. Neuroscience \& Biobehavioral Reviews, 34, 7-22. doi: 10.1016/j.neubiorev.2009.06.008

Kasai, K., Yamasue, H., Gilbertson, M. W., Shenton, M. E., Rauch, S. L., \& Pitman, R. K. (2008). Evidence for acquired pregenual anterior cingulate gray matter loss from a twin study of combat-related posttraumatic stress disorder. Biological Psychiatry, 63, 550-556. doi: 10.1016/ j.biopsych.2007.06.022

Kolassa, I.-T., \& Elbert, T. (2007). Structural and functional neuroplasticity in relation to traumatic stress. Current Directions in Psychological Science, 16, 321-325. doi: 10.1111/j.1467-8721.2007.00529.x

Kolassa, I. T., Wienbruch, C., Neuner, F., Schauer, M., Ruf, M., Odenwald, M., \& Elbert, T. (2007). Altered oscillatory brain dynamics after repeated traumatic stress. BMC Psychiatry, 7, 56. doi: 10.1186/1471244X-7-56

Liberzon, I., \& Martis, B. (2006). Neuroimaging studies of emotional responses in PTSD. Annals of the New York Academy of Sciences, 1071 87-109. doi: 10.1196/annals.1364.009

Lindauer, R. J., Vlieger, E. J., Jalink, M., Olff, M., Carlier, I. V., Majoie, C. B., . . Gersons, B. P. (2004). Smaller hippocampal volume in Dutch police officers with posttraumatic stress disorder. Biological Psychiatry, 56, 356-363. doi: 10.1016/j.biopsych.2004.05.021 
McNally, R. J. (2006). Cognitive abnormalities in post-traumatic stress disorder. Trends in Cognitive Sciences, 10, 271-277, doi: 10.1016 j.tics.2006.04.007

Miller, G. A., \& Chapman, J. P. (2001). Misunderstanding analysis of covariance. Journal of Abnormal Psychology, 110, 40-48. doi:.10.1037/ 0021-843X.110.1.40

Mohanakrishnan Menon, P., Nasrallah, H. A., Lyons, J. A., Scott, M. F., \& Liberto, V. (2003). Single-voxel proton MR spectroscopy of right versus left hippocampi in PTSD. Psychiatry Research: Neuroimaging, 123. 101-108. doi: 10.1016/S0925-4927(03)00044-1

O'Donnell, M. L., Creamer, M., \& Pattison, P. (2004). Posttraumatic stress disorder and depression following trauma: Understanding comorbidity. American Journal of Psychiatry, 161, 1390-1396. doi: 10.1176/ appi.ajp.161.8.1390

Oldfield, R. C. (1971). The assessment and analysis of handedness: The Edinburgh inventory. Neuropsychologia, 9, 97-113. doi: 10.1016/00283932(71)90067-4

Osuch, E. A., Benson, B., Geraci, M., Podell, D., Herscovitch, P. McCann, U. D., \& Post, R. M. (2001). Regional cerebral blood flow correlated with flashback intensity in patients with posttraumatic stress disorder. Biological Psychiatry, 50, 246-253. doi: 10.1016/S00063223(01)01107-6

Phan, K. L., Wager, T., Taylor, S. F., \& Liberzon, I. (2002). Functional neuroanatomy of emotion: A meta-analysis of emotion activation studies in PET and fMRI. Neurolmage, 16, 331-348. doi: 10.1006/ nimg.2002.1087

Pinheiro, J., \& Bates, D. (2000). Mixed-effects models in S and S-PLUS. New York, NY: Springer-Verlag. doi: 10.1007/978-1-4419-0318-1

Pinheiro, J., Bates, D., DebRoy, S., Sarkar, D., \& R Core Team. (2008). NLME: Linear and nonlinear mixed effects models. (Version R package version 3.1-90)

Pruessner, J. C., Li, L. M., Serles, W., Pruessner, M., Collins, D. L., Kabani, N., .. Evans, A. C. (2000). Volumetry of hippocampus and amygdala with high-resolution MRI and three-dimensional analysis software: Minimizing the discrepancies between laboratories. Cerebral Cortex 10, 433-442. doi: $10.1093 /$ cercor/10.4.433

R Development Core Team. (2007). R: A language and environment for statistical computing. Vienna, Austria. http://cran.r-project.org/

Rasch, B., Spalek, K., Buholzer, S., Luechinger, R., Boesiger, P., Papassotiropoulos, A., \& deQuervain, D. J. (2009). A genetic variation of the noradrenergic system is related to differential amygdala activation during encoding of emotional memories. Proceedings of the National Academy of Sciences of the US A, 106, 19191-19196. doi: 10.1073/ pnas.0907425106

Rolls, E. T., Xiang, J., \& Franco, L. (2005). Object, space, and object-space representations in the primate hippocampus. Journal of Neurophysiology, 94, 833-844. doi: 10.1152/jn.01063.2004

Rooney, W. D., Johnson, G., Li, X., Cohen, E. R., Kim, S. G., Ugurbil, K., \& Springer, C. S., Jr. (2007). Magnetic field and tissue dependencies of human brain longitudinal $1 \mathrm{H} 2 \mathrm{O}$ relaxation in vivo. Magnetic Resonance in Medicine, 57, 308-318. doi: $10.1002 / \mathrm{mrm} .21122$

Sapolsky, R. M., Uno, H., Rebert, C. S., \& Finch, C. E. (1990). Hippocampal damage associated with prolonged glucocorticoid exposure in primates. Journal of Neuroscience, 10, 2897-2902.

Sar, V., Tutkun, H., Alyanak, B., Bakim, B., \& Baral, I. (2000). Frequency of dissociative disorders among psychiatric outpatients in Turkey. Comprehensive Psychiatry, 41, 216-222. doi: 10.1016/S0010440X(00)90050-6

Schauer, M., Neuner, F., \& Elbert, T. (2011). Narrative exposure therapy (2nd ed.). Göttingen, Germany: Hogrefe \& Huber.

Schmahl, C., Berne, K., Krause, A., Kleindienst, N., Valerius, G., Vermetten, E., \& Bohus, M. (2009). Hippocampus and amygdala volumes in patients with borderline personality disorder with or without posttraumatic stress disorder. Journal of Psychiatry \& Neuroscience, 34, 289 295.
Schuff, N., Neylan, T. C., Fox-Bosetti, S., Lenoci, M., Samuelson, K. W., Studholme, C., ... Weiner, M. H. (2008). Abnormal N-acetylaspartate in hippocampus and anterior cingulate in posttraumatic stress disorder. Psychiatry Research: Neuroimaging, 162, 147-157. doi: 10.1016/ j.pscychresns.2007.04.011

Schuff, N., Neylan, T. C., Lenoci, M. A., Du, A. T., Weiss, D. S., Marmar, C. R., \& Weiner, M. W. (2001). Decreased hippocampal $\mathrm{N}$-acetylaspartate in the absence of atrophy in posttraumatic stress disorder. Biological Psychiatry, 50, 952-959. doi: 10.1016/S00063223(01)01245-8

Segonne, F., Dale, A. M., Busa, E., Glessner, M., Salat, D., Hahn, H. K. \& Fischl, B. (2004). A hybrid approach to the skull stripping problem in MRI. Neurolmage, 22, 1060-1075. doi: 10.1016/ j.neuroimage.2004.03.032

Sheehan, D. V., Lecrubier, Y., Sheehan, K. H., Amorim, P., Janavs, J., \& Weiller, E. (1998). The Mini-International Neuropsychiatric Interview (M.I.N.I.): The development and validation of a structured diagnostic psychiatric interview for DSM-IV and ICD-10. Journal of Clinical Psychiatry, 59 (Suppl 20) 22-33; quiz 34-57.

Shin, L. M., Shin, P. S., Heckers, S., Krangel, T. S., Macklin, M. L., Orr, S. P., . . \& \& Rauch, S. L. (2004). Hippocampal function in posttraumatic stress disorder. Hippocampus, 14, 292-300. doi: 10.1002/hipo.10183

Stanisz, G. J., Odrobina, E. E., Pun, J., Escaravage, M., Graham, S. J., Bronskill, M. J., \& Henkelman, R. M. (2005). T1, T2 relaxation and magnetization transfer in tissue at 3T. Magnetic Resonance in Medicine 54, 507-512. doi: 10.1002/mrm.20605

Teicher, M. H., Andersen, S. L., Polcari, A., Anderson, C. M., Navalta, C. P., \& Kim, D. M. (2003). The neurobiological consequences of early stress and childhood maltreatment. Neuroscience \& Biobehavioral Reviews, 27, 33-44. doi: 10.1016/S0149-7634(03)00007-1

Vythilingam, M., Luckenbaugh, D. A., Lam, T., Morgan, C. A., 3rd, Lipschitz, D., Charney, D. S., ... Southwick, S. M. (2005). Smalle head of the hippocampus in Gulf War-related posttraumatic stress disorder. Psychiatry Research: Neuroimaging, 139, 89-99. doi: 10.1016/ j.pscychresns.2005.04.003

Woodward, S. H., Kaloupek, D. G., Grande, L. J., Stegman, W. K., Kutter, C. J., Leskin, L. Eliez, S. (2009). Hippocampal volume and declarative memory function in combat-related PTSD. Journal of the International Neuropsychological Society: JINS, 15, 830-839. doi: 10.1017/ S1355617709990476

Woodward, S. H., Kaloupek, D. G., Streeter, C. C., Kimble, M. O., Reiss, A. L., Eliez, S., ... Arsenault, N. J. (2006). Hippocampal volume, PTSD, and alcoholism in combat veterans. American Journal of Psychiatry, 163, 674-681. doi: 10.1176/appi.ajp.163.4.674

Zaidi, L. Y., \& Foy, D. W. (1994). Childhood abuse experiences and combat-related PTSD. Journal of Traumatic Stress, 7, 33-42. doi: $10.1002 /$ jts. 2490070105

Table S1: Volumetric and spectroscopic measures. 